\title{
Effect of Salvia miltiorrhiza and ligustrazine injection on myocardial ischemia/reperfusion and hypoxia/reoxygenation injury
}

\author{
WENDONG HUANG ${ }^{1,2}$, YONGFEI YANG ${ }^{1}, \mathrm{ZHI}_{\mathrm{ZENG}}{ }^{1}, \mathrm{MEILING} \mathrm{SU}^{1}, \mathrm{QI} \mathrm{GAO}^{1}$ and BANGHAO ZHU ${ }^{1}$ \\ ${ }^{1}$ Department of Pharmacology, Cardiac and Cerebral Vascular Research Center, Zhongshan School of Medicine, \\ Sun Yat-sen University, Guangzhou, Guangdong 510080; ${ }^{2}$ Department of Pharmacy, \\ Maoming People's Hospital, Maoming, Guangdong 525000, P.R. China
}

Received August 11, 2015; Accepted August 17, 2016

DOI: $10.3892 / \mathrm{mmr} .2016 .5822$

\begin{abstract}
Salvia miltiorrhiza and ligustrazine are traditional Chinese medicines that have been used in combination for treatment of cardiovascular disease, including coronary heart disease, cardiac angina and atherosclerosis in Asia, in particular, China. The present study aimed to determine the effect of S. miltiorrhiza and ligustrazine injection (SLI) on myocardial ischemia/reperfusion (I/R) and hypoxia/reoxygenation $(\mathrm{H} / \mathrm{R})$ injuries via the Akt serine/threonine kinase (Akt)-endothelial nitric oxide synthase (eNOS) signaling pathway. Male Sprague-Dawley rats were randomly assigned into six groups: i) Sham group; ii) I/R group; iii) Low-SLI group (6.8 mg/kg/day, i.p.); iv) Medium-SLI group (20.4 mg/kg/day, i.p.); v) High-SLI group (61.2 mg/kg/day, i.p.); vi) verapamil group (6 mg/kg/day, i.p.). Prior to surgery, the aforementioned groups were pretreated with a homologous drug once per day for 3 days. The effect of SLI following 35 min coronary artery occlusion and $2 \mathrm{~h}$ reperfusion was evaluated by determining infarctsize, hemodynamics, biochemical values and histological observations. Additionally, cell viability, caspase-3 expression, B cell leukemia/lymphoma-2 (Bcl-2)/Bcl-2-associated $\mathrm{X}$ protein (Bax) ratio, phosphorylated (p-)Akt and p-eNOS were also investigated following $2 \mathrm{~h}$ simulated ischemia and $2 \mathrm{~h}$ simulated reperfusion in $\mathrm{H} 9 \mathrm{C} 2$ cardiomyocyte cells. Pretreatment with SLI significantly improved cardiac function in a dose-dependent manner and reduced myocardial infarct size, creatine kinase, lactate dehydrogenase and malondialdehyde levels in blood serum. Additionally, myocardial
\end{abstract}

Correspondence to: Professor Banghao Zhu, Department of Pharmacology, Cardiac and Cerebral Vascular Research Center, Zhongshan School of Medicine, Sun Yat-sen University, 74 Zhongshan Road 2, Guangzhou, Guangdong 510080, P.R. China E-mail: zhubh@mail.sysu.edu.cn

Key words: Salvia miltiorrhiza, myocardial ischemia/reperfusion, ligustrazine, hypoxia/reoxygenation, cardioprotective effect histopathology changes in the rat model were also alleviated in SLI treatment groups. The present in vitro study revealed that treatment with SLI reduced the apoptotic rate of $\mathrm{H} 9 \mathrm{C} 2$ cells by inhibiting the activation of caspase- 3 and increasing the $\mathrm{Bcl}-2 / \mathrm{Bax}$ ratio. The effect of SLI was associated with increased phosphorylation of the survival kinase Akt at Ser473 and its downstream target eNOS following H/R. The present study determined that SLI may alleviate I/R injury in cardiomyocytes and inhibit apoptosis in rats by the activation of the Akt-eNOS signaling pathway, and downregulation of the expression levels of proapoptotic factors, including caspase-3.

\section{Introduction}

Cardiovascular diseases have the highest morbidity rate worldwide, including angina, coronary heart disease, hypertension and myocardial infarction (MI) (1). A previous study determined that by the year 2020, MI may be the primary cause of mortality worldwide (2). MI occurs when coronary blood flow is inadequate, leading to cardiac dysfunction, arrhythmias and sudden mortality (3). Prevention of myocardial ischemia/reperfusion (I/R) injury is vital for successful coronary heart disease surgery and recovery (4). It is important to develop novel treatments to inhibit or reduce disease process and gain time for subsequent treatment. Traditional Chinese Medicine has gained increasing attention for the treatment of various diseases. The use of Chinese medicine for ventricular remodeling treatments has been investigated previously; however, it has been demonstrated that using Traditional Chinese Medicine may affect the occurrence and development of ventricular remodeling in a number of aspects, for example proliferation and apoptosis (5). Additionally, previous studies have determined that specific agents, including statins, angiotensin converting enzyme inhibitors and angiotensin II receptor inhibitors, may improve ventricular remodeling by increasing cell apoptosis and reducing cell proliferation in smooth muscle cells in hypertensive animal models $(6,7)$.

Danshen is a Traditional Chinese Medicine and is primarily used as treatment for cardiovascular diseases, including angina pectoris, MI and stroke (8). Previous in vitro and in vivo studies revealed that danshen exerts various 
pharmacological effects, including relaxation of the coronary artery, anticoagulation, reduction of myocardial I/R injury and antiarrhythmic effects (9-11). By contrast, ligustrazine has been previously reported to increase coronary blood flow and systemic circulation by protecting mitochondria and improving energy metabolism, scavenging oxygen free radicals in order to inhibit lipid peroxidation, inhibition of apoptosis and protection of myocardial cells, reducing the inflammatory reaction, mitigating cell injury, and protecting myocardial cells (12-14). Our previous study demonstrated that a compound of Salvia miltiorrhiza and ligustrazine may act synergistically on the cardiovascular system in rats (15). The present study determined the effect of S. miltiorrhiza and ligustrazine injection (SLI) (ratio of S. miltiorrhiza to ligustrazine was 1:50) on myocardial I/R injury and investigated the underlying molecular mechanism.

\section{Materials and methods}

Animals and treatment. SLI was obtained from Jilin Sichang Pharmaceutical Co. Ltd. (Jilin, China). A total of 80 male Sprague-Dawley rats (weight, 250-280 g; age, 8 weeks) were purchased from the Animal Research Center of Sun Yat-sen University (Guangzhou, China). The animal procedures were performed according to a protocol approved by the Central Animal Facility of Sun Yat-sen University (no. scxk 2011-0029). All animals were housed in a room with a $12 \mathrm{~h}$ light-dark cycle at a temperature $22-24^{\circ} \mathrm{C}$ and humidity of $50-60 \%$, with standard laboratory rodent chow and water ad libitum. Rats were euthanized by overdose of anesthetic.

Myocardial I/R injury model of rats. The cardiac I/R surgery was performed on the rats, as previously described with certain modifications (16). The rats were anaesthetized via intraperitoneal injection of pentobarbital sodium $(60 \mathrm{mg} / \mathrm{kg}$ body weight; Merck Millipore, Darmstadt, Germany), using a heating pad to maintain animal body temperature at $37^{\circ} \mathrm{C}$ during surgery. A tracheotomy was performed and the trachea was cannulated with a mechanical ventilator (Alcott Biology, Shanghai, China) to establish artificial respiration using oxygen at a frequency of $70-80$ breaths $/ \mathrm{min}$ and tidal volume of $15 \mathrm{ml} / \mathrm{kg}$. The chest was opened at the left fourth intercostal space to expose the heart using a left thoracotomy. The left anterior descending (LAD) artery was passed using a 6-0 silk suture, its ends were threaded through a tube in order to form a snare. Successful LAD artery occlusion was confirmed by electrocardiogram (ECG) to identify injury and myocardial cyanosis. The heart was subjected to regional ischemia for $35 \mathrm{~min}$, followed by coronary reperfusion for $2 \mathrm{~h}$ following the release of the tube.

Treatment groups. The animals were randomly divided into the following six treatment groups (n=8/group): i) Sham, ii) I/R, iii) Low (L-)SLI, iv) Medium (M-)SLI, v) High (H-) SLI; vi) verapamil (Ver; Shandong Xinhua Pharmaceutical Co., Ltd., Zibo, China) groups. The animals in the sham group underwent identical surgical procedures without LAD ligation. In the I/R group, the coronary artery was occluded and vehicle (veh) treatment was administered. For the SLI treatment groups, SLI was dissolved in saline at a final concentration of $6.8,20.4$ and $61.2 \mathrm{mg} / \mathrm{kg}(0.133: 6.667,0.4: 20$ and $1.2: 60 \mathrm{mg} / \mathrm{kg} \mathrm{S}$. miltiorrhiza:ligustrazine) for the L-, M- and H-SLI groups, respectively, administered by intraperitoneal injection once a day for 3 days prior to surgery. The rats in the Ver group were treated with $6 \mathrm{mg} / \mathrm{kg}$ Ver by intraperitoneal injection once/day for 3 days.

Hemodynamics and ECG in rats. The right carotid artery was isolated and a catheter was inserted. A biotic signal system (PowerLab; AD Instruments, Sydney, Australia) was used to record various cardiac functions of the rats in the different groups, including the left ventricular systolic pressure (LVSP), left ventricular diastolic pressure (LVDP), first derivative of left ventricular pressure ( $\pm \mathrm{dp} / \mathrm{dt})$, heart rate $(\mathrm{HR})$ and ECG.

Determination of lactate dehydrogenase $(L D H)$, superoxide dismutase (SOD), creatine kinase $(C K)$ and malondialdehyde (MDA) activity in blood serum. After $3 \mathrm{~h}$ reperfusion, blood samples were collected from the abdominal aortic artery and blood serum was stored at $-80^{\circ} \mathrm{C}$ until analysis. The levels of LDH, SOD, CK and MDA were determined using a Hitachi 7180 automated biochemical analyzer system (Diamond Diagnostics, Holliston, MA, USA).

Determination of the infarct size. The size of the cardiac infarct was determined using a triphenyl tetrazolium chloride (TTC) staining method, as previously described $(17,18)$. Rats were sacrificed by intraperitoneal injectino of an overdose of pentobarbital sodium anesthesia $(300 \mathrm{mg} / \mathrm{kg}$ body weight) subsequent to the collection of the serum samples, the heart was excised and weighed immediately. The left ventricle was sliced parallel to the atrioventricular groove into five sections with a thickness of $2-3 \mathrm{~mm}$. The myocardial slices were incubated for $15 \mathrm{~min}$ at $37^{\circ} \mathrm{C}$ in $1 \% \mathrm{TTC}$ solution in phosphate-buffered saline. Next, images of the slices were captured. The infarct area was analyzed using Image-Pro Plus version 5.0 software (Media Cybernetics, Inc., Rockville, MD, USA). Infarct size was expressed as a percentage, calculated by dividing the infarct mass of the left ventricle by its total mass. This was analyzed using Image J version 1.26 (National Institutes of Health, Bethesda, MD, USA).

Histopathological examination of the cardiac tissues. Cardiac tissues were obtained below ligation to the apex area of the heart and fixed in 10\% buffered formalin. Tissue sections $(\sim 7 \mu \mathrm{m})$ were prepared from the paraffin-embedded tissues. The tissues were stained with hematoxylin and eosin. The extent of the tissue injury was determined using a microscope at a magnification $\mathrm{x} 400$.

Cell culture and hypoxia/reoxygenation $(H / R)$ injury. The $\mathrm{H} 9 \mathrm{C} 2$ rat cardiomyocyte cell line was obtained from the China Infrastructure of Cell Line Resources (Chinese Academy of Medical Sciences, Beijing, China). H9C2 cells were cultured in Dulbecco's modified Eagle's medium (Gibco; Thermo Fisher Scientific, Inc., Waltham, MA, USA) with 10\% fetal calf serum (PAA; GE Healthcare Life Sciences, Pittsburgh, PA, USA) and $1 \%$ of penicillin-streptomycin, and were incubated at $37^{\circ} \mathrm{C}$, with $5 \% \mathrm{CO}_{2}$. New medium was applied 
A

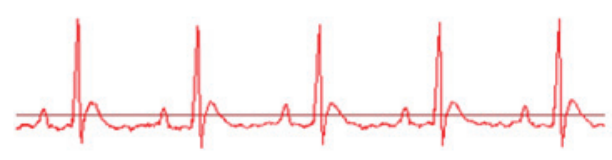

C

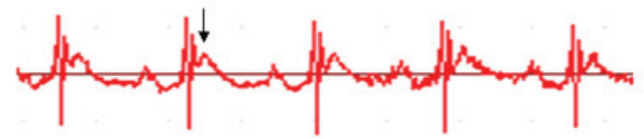

$\mathbf{E}$

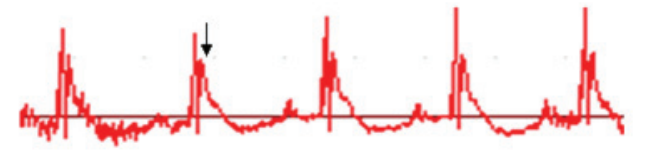

B

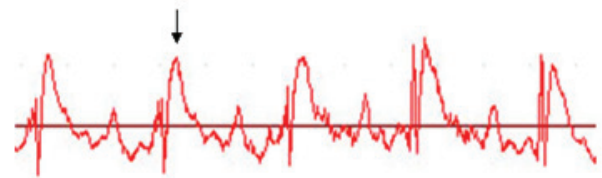

D

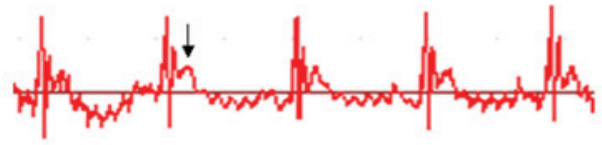

$\mathbf{F}$

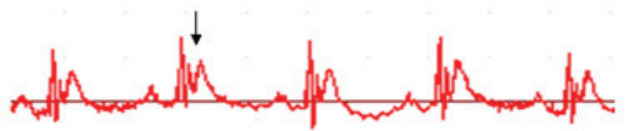

Figure 1. Effect of SLI on electrocardiogram changes of myocardial infarction in (A) Sham, (B) I/R, (C) L-SLI, (D) M-SLI, (E) H-SLI and (F) verapamil groups $2 \mathrm{~h}$ after reperfusion in rats. ST segments indicated by black arrows. SLI, Salvia miltiorrhiza and ligustrazine injection; I/R, ischemia/reperfusion; L-, low-; M-, medium-; H-, high-.

every 2-3 days. The cells were subjected to experimental procedures at $80-90 \%$ confluence.

The H/R injury model was implemented, as previously described (1-3). Briefly, H9C2 cells were exposed to ischemia by replacing the medium with an 'hypoxic buffer' (0.9 mM NaH${ }_{2} \mathrm{PO}_{4}, 6.0 \mathrm{mM} \mathrm{NaHCO} 3,98.5 \mathrm{mM} \mathrm{NaCl}$, $1.2 \mathrm{mM} \mathrm{MgSO}_{4}, 10 \mathrm{mM} \mathrm{KCl}, 1.8 \mathrm{mM} \mathrm{CaCl}_{2}, 40 \mathrm{mM}$ sodium lactate, $20 \mathrm{mM}$ HEPES; pH 6.8). The cells were incubated in a hypoxic chamber at $37^{\circ} \mathrm{C}$ for $2 \mathrm{~h}$ in a humidified atmosphere of $5 \% \mathrm{CO}_{2}$ and $95 \%$ nitrogen. Following $2 \mathrm{~h}$ of hypoxic conditions, the cells were exposed to reoxygenation by replacing the medium with a 'reoxygenation buffer' (20.0 mM NaHCO $3,0.9 \mathrm{mM} \mathrm{NaH}{ }_{2} \mathrm{PO}_{4}, 1.8 \mathrm{mM} \mathrm{CaCl}_{2}$, $1.2 \mathrm{mM} \mathrm{MgSO}_{4}, 20.0 \mathrm{mM}$ HEPES, $5.0 \mathrm{mM} \mathrm{KCl}, 129.5 \mathrm{mM}$ $\mathrm{NaCl}, 5.5 \mathrm{mM}$ glucose; $\mathrm{pH}$ 7.4) in a standard incubator for $3 \mathrm{~h}$. The cells in the control group were cultured with normal Tyrode's solution (pH 7.4; Beijing Reagant Biological Technology Co., Ltd., Beijing, China) in a standard incubator for $5 \mathrm{~h}$ (4). A total of five treatment groups were established: i) Control; ii) H/R; iii) L-SLI; iv) M-SLI; and v) H-SLI. SLI was administered prior to 2-h H/R injury within the normal culture medium at $37^{\circ} \mathrm{C}$.

Cell viability assay. Cell viability was determined using a Cell Counting Kit (CCK)-8 assay (Beyotime Institute of Biotechnology, Inc., Guangzhou, China), according to the manufacturer's protocol. H9C2 cells were seeded at a density of $5 \times 10^{4}$ cells/well into 96 -well plates. Following the aforementioned treatments, the cells were exposed to $10 \mu \mathrm{l}$ CCK- 8 solution for $2 \mathrm{~h}$ and the absorbance was determined at $450 \mathrm{~nm}$ using a microplate reader (BioTek Instruments, Inc., Winooski, VT, USA).

Western blot analysis. H9C2 cells were lysed in lysis buffer (Beyotime Institute of Biotechnology, Inc.) containing protease inhibitor cocktail (Merck Millipore) on ice for $30 \mathrm{~min}$. Cellular proteins were collected using a cell scraper and the lysates were centrifuged at $4{ }^{\circ} \mathrm{C}$ for $15 \mathrm{~min}$ at $10,000 \times \mathrm{g}$. A BCA Protein Assay kit (Beyotime Institute of Biotechnology, Inc.) was used to determine protein concentrations. Equal quantities of protein $(2 \mu \mathrm{g} / \mu \mathrm{l})$ were separated on $8 \%$ sodium dodecyl sulphate-polyacrylamide gels and transferred onto a polyvinylidene difluoride membrane (EMD Millipore, Billerica, MA, USA). The membranes were blocked with $5 \%$ non-fat dry milk for $30 \mathrm{~min}$ at room temperature. Following blocking, the membranes were incubated with the primary antibodies against rabbit $\mathrm{IgG}$ p-eNOS/eNOS (1:1,000; BD Biosciences, Franklin Lakes, NJ, USA), rabbit IgG p-Akt/Akt (1:1,000; BD Biosciences), Bcl-2 (1:1,000; Cell Signaling Technology, Inc., Danvers, MA, USA), Bax (1:2,000; Cell Signaling Technology, Inc.) and caspase-3 (1:3,000; Cell Signaling Technology, Inc.) overnight at $4^{\circ} \mathrm{C}$. The membranes were washed with Tris-buffered saline containing Tween 20 and were subsequently incubated with secondary antibodies: Anti-mouse (IgG; 1:5,000) or horseradish peroxidase-conjugated anti-rabbit $(\mathrm{IgG} ; 1: 1,000)$ for $1 \mathrm{~h}$ (all obtained from Cell Signaling Technology, Inc.). The membranes were washed as before. $\beta$-actin (IgG; 1:3,000; Cell Signaling Technology, Inc.) was used as the internal reference. The protein bands were obtained using an enhanced chemiluminescence system (Beyotime Institute of Biotechnology, Inc.). The band density was scanned and quantified using Image-J software (National Institutes of Health).

Statistical analysis. The data are expressed as the mean \pm standard deviation. Statistical comparisons between groups were performed using one-way analysis of variance. SPSS software, version 19 (SPSS, Inc., Chicago, IL, USA). $\mathrm{P}<0.05$ was considered to indicate a statistically significant difference.

\section{Results}

SLI improves cardiac function. Changes in the hemodynamic data that occurred during the experiments are presented in Tables I-III. No difference in the baseline was observed between the different groups. Additionally, no significant difference was identified during the experiment in the sham group (Table I). Pretreatment with all doses of SLI and Ver significantly increased LVSP $(\mathrm{P}<0.05$; Table I) and the $+\mathrm{dp} / \mathrm{dt}$ $\max (\mathrm{P}<0.05$; Table II) compared with the I/R group. LVDP 
Table I. Effects of SLI on LVSP and LVDP of myocardial infarction in rats.

\begin{tabular}{|c|c|c|c|c|c|c|}
\hline \multirow[b]{2}{*}{ Group } & \multicolumn{3}{|c|}{ LVSP (mmHg) } & \multicolumn{3}{|c|}{ LVDP (mmHg) } \\
\hline & Baseline & Ischemia & Reperfusion & Baseline & Ischemia & Reperfusion \\
\hline Sham & $149.81 \pm 4.10$ & $150.26 \pm 5.50$ & $147.03 \pm 4.90$ & $24.49 \pm 2.10$ & $23.81 \pm 2.50$ & $24.52 \pm 2.90$ \\
\hline $\mathrm{I} / \mathrm{R}$ & $136.32 \pm 11.70$ & $96.78 \pm 13.87^{c}$ & $91.73 \pm 13.79^{c}$ & $23.88 \pm 5.19$ & $15.92 \pm 4.10^{c}$ & $17.75 \pm 4.93$ \\
\hline L-SLI & $139.22 \pm 7.22$ & $99.08 \pm 8.23^{\mathrm{a}}$ & $100.84 \pm 7.79^{\mathrm{a}}$ & $22.45 \pm 6.22$ & $17.34 \pm 5.23$ & $16.83 \pm 6.97$ \\
\hline M-SLI & $142.48 \pm 16.72$ & $106.85 \pm 22.15^{\mathrm{a}}$ & $107.05 \pm 11.86^{\mathrm{a}}$ & $25.72 \pm 3.44$ & $15.05 \pm 4.97$ & $17.15 \pm 4.60$ \\
\hline H-SLI & $140.30 \pm 18.23$ & $103.73 \pm 26.87^{\mathrm{a}}$ & $113.90 \pm 11.12^{b}$ & $24.88 \pm 3.19$ & $25.59 \pm 4.96^{\mathrm{b}}$ & $26.59 \pm 4.42^{\prime}$ \\
\hline Ver & $152.57 \pm 5.23$ & $114.30 \pm 6.15^{\mathrm{b}}$ & $111.20 \pm 4.78^{\mathrm{b}}$ & $22.07 \pm 5.32$ & $20.60 \pm 4.15^{\mathrm{a}}$ & $25.60 \pm 4.78^{\mathrm{b}}$ \\
\hline
\end{tabular}

Data are presented as the mean \pm standard deviation $\left(\mathrm{n}=8 ;{ }^{\mathrm{a}} \mathrm{P}<0.05,{ }^{\mathrm{b}} \mathrm{P}<0.01 \mathrm{vs}\right.$. I/R group; ${ }^{\mathrm{c}} \mathrm{P}<0.01 \mathrm{vs}$. sham group). LSVP, left ventricular systolic pressure; LVDP, left ventricular diastolic pressure; SLI, Salvia miltiorrhiza and ligustrazine injection; I/R, ischemia/reperfusion; L-, low-; M-, medium-; H-, high-; Ver, verapamil.

Table II. Effects of SLI on $+\mathrm{dp} / \mathrm{dt}(\max )$ and -dp/dt (max) of myocardial infarction in rats.

\begin{tabular}{|c|c|c|c|c|c|c|}
\hline \multirow[b]{2}{*}{ Group } & \multicolumn{3}{|c|}{$+\mathrm{dp} / \mathrm{dt}(\max )(\mathrm{mmHg} / \mathrm{ms})$} & \multicolumn{3}{|c|}{$-\mathrm{dp} / \mathrm{dt}(\max )(\mathrm{mmHg} / \mathrm{ms})$} \\
\hline & Baseline & Ischemia & Reperfusion & Baseline & Ischemia & Reperfusion \\
\hline Sham & $1.31 \pm 0.23$ & $1.29 \pm 0.18$ & $1.33 \pm 0.22$ & $1.28 \pm 0.12$ & $1.33 \pm 0.28$ & $1.36 \pm 0.27$ \\
\hline $\mathrm{I} / \mathrm{R}$ & $1.26 \pm 0.28$ & $0.80 \pm 0.21^{\mathrm{c}}$ & $0.85 \pm 0.29^{c}$ & $1.13 \pm 0.17$ & $0.65 \pm 0.30^{\mathrm{c}}$ & $0.73 \pm 0.24^{c}$ \\
\hline L-SLI & $1.20 \pm 0.26$ & $0.92 \pm 0.21^{\mathrm{a}}$ & $1.02 \pm 0.27^{\mathrm{a}}$ & $1.25 \pm 0.26$ & $1.27 \pm 0.31^{\mathrm{b}}$ & $1.19 \pm 0.22^{b}$ \\
\hline M-SLI & $1.12 \pm 0.24$ & $1.09 \pm 0.25^{\mathrm{a}}$ & $1.06 \pm 0.20^{\mathrm{a}}$ & $1.02 \pm 0.11$ & $1.02 \pm 0.15^{\mathrm{b}}$ & $1.08 \pm 0.22^{\mathrm{a}}$ \\
\hline H-SLI & $1.27 \pm 0.24$ & $1.45 \pm 0.20^{\mathrm{b}}$ & $1.34 \pm 0.22^{\mathrm{b}}$ & $1.23 \pm 0.11$ & $1.11 \pm 0.06^{\mathrm{b}}$ & $1.38 \pm 0.28^{b}$ \\
\hline Ver & $1.20 \pm 0.25$ & $1.08 \pm 0.18^{\mathrm{a}}$ & $1.17 \pm 0.18^{\mathrm{a}}$ & $1.25 \pm 0.28$ & $0.96 \pm 0.32$ & $1.20 \pm 0.29^{b}$ \\
\hline
\end{tabular}

Data are presented as the mean \pm standard deviation ( $\mathrm{n}=8 ;{ }^{\mathrm{a}} \mathrm{P}<0.05,{ }^{\mathrm{b}} \mathrm{P}<0.01 \mathrm{vs}$. I/R group; ${ }^{\mathrm{c}} \mathrm{P}<0.01 \mathrm{vs}$. sham group). SLI, Salvia miltiorrhiza and ligustrazine injection; I/R, ischemia/reperfusion; L-, low-; M-, medium-; H-, high-; Ver, verapamil.

$(\mathrm{P}<0.05$; Table I) and the -dp/dt max $(\mathrm{P}<0.05$; Table II) were significantly decreased in response to pretreatment with SLI and Ver. However, no significant difference was observed in the heart rate between the different groups during coronary artery occlusion and reperfusion (Table III).

Effect of SLI on the ST segment of the ECG in I/R rats. Changes in ECG, including the degrees of T wave and ST segment, may be used to evaluate MI in the animals. The present study determined that $\mathrm{T}$ waves were consistent in the sham group rats. The ST-segments were elevated in the I/R group following the ischemia surgery. The ST-segments in the remaining groups were reduced compared with the I/R group following reperfusion (data not shown; Fig. 1).

Effect of SLI on LDH, CK, SOD and MDA levels in blood serum. In order to determine whether $S$. miltiorrhiza and ligustrazine may reduce the damage of cardiomyocytes following I/R, characterized by cell membrane disruption and cell content release, the activities of CK, LDH, SOD and MDA in blood serum were determined at the end of reperfusion. As shown in Fig. 2, the levels of CK, LDH and MDA were increased in the I/R group and SOD levels were significantly
Table III. Effects of SLI on HR of myocardial infarction in rats.

\begin{tabular}{lccc}
\hline & \multicolumn{3}{c}{ HR (beats/min) } \\
\cline { 2 - 4 } Group & Baseline & Ischemia & Reperfusion \\
\hline Sham & $376.42 \pm 32.69$ & $368.18 \pm 25.75$ & $372.63 \pm 22.64$ \\
I/R & $399.83 \pm 52.75$ & $363.33 \pm 27.46$ & $374.00 \pm 32.30$ \\
L-SLI & $385.50 \pm 63.78$ & $371.00 \pm 64.11$ & $389.25 \pm 27.97$ \\
M-SLI & $387.75 \pm 23.21$ & $353.25 \pm 47.32$ & $395.00 \pm 76.15$ \\
H-SLI & $392.00 \pm 36.06$ & $341.67 \pm 20.23$ & $322.67 \pm 60.80$ \\
Ver & $368.50 \pm 19.09$ & $300.00 \pm 75.54$ & $334.00 \pm 77.78$ \\
\hline
\end{tabular}

Data are presented as the mean \pm standard deviation $(n=8)$. SLI, Salvia miltiorrhiza and ligustrazine injection; HR, heart rate; I/R, ischemia/reperfusion; L-, low-; M-, medium-; H-, high-; Ver, verapamil.

decreased in the $\mathrm{I} / \mathrm{R}$ group compared with the sham group $(\mathrm{P}<0.05$; Fig. 2B). Pretreatment with SLI and Ver significantly decreased the levels of CK, LDH and MDA in blood serum 
A
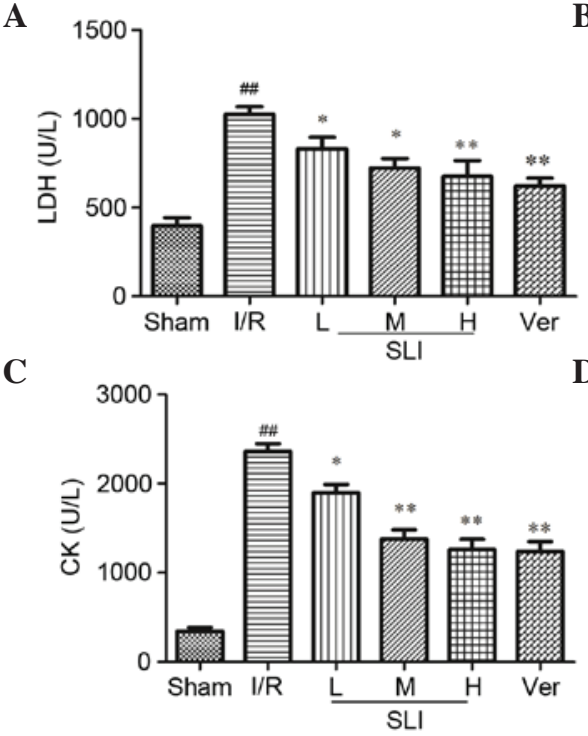

B
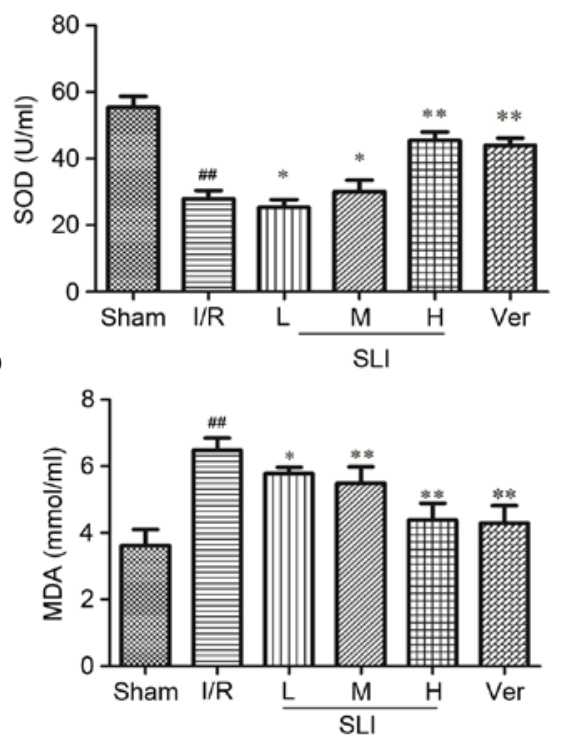

Figure 2. Effects of SLI on the activity of various molecules. The (A) LDH, (B) SOD, (C) CK and (D) MDA activity in blood serum of rats was measured using an automated biochemical analyzer system following myocardial I/R injury. LDH, lactate dehydrogenase; SOD, superoxide dismutase; CK, creatine kinase; MDA, malondialdehyde; SLI, Salvia miltiorrhiza and ligustrazine injection; I/R, ischemia/reperfusion; L-, low-; M-, medium-; H-, high-; Ver, verapamil. Data are presented as the mean \pm standard deviation $\left(\mathrm{n}=8 ;{ }^{*} \mathrm{P}<0.05,{ }^{* *} \mathrm{P}<0.01\right.$ vs. I/R group; ${ }^{\# \#} \mathrm{P}<0.01$ vs. sham group).

\section{A}

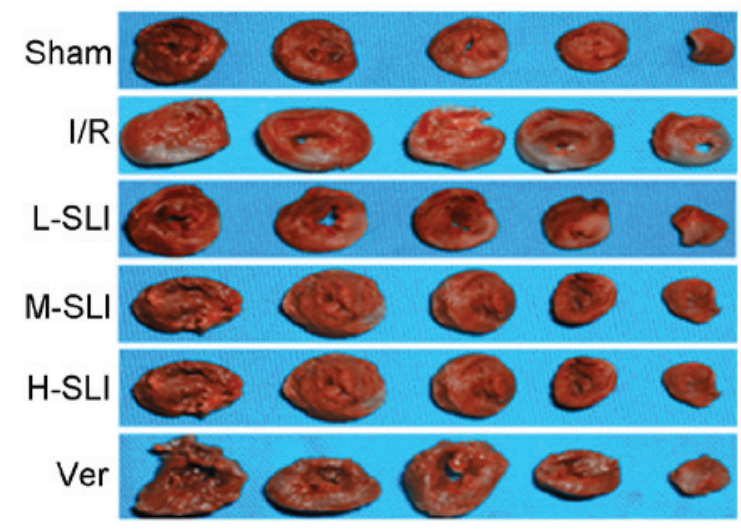

B

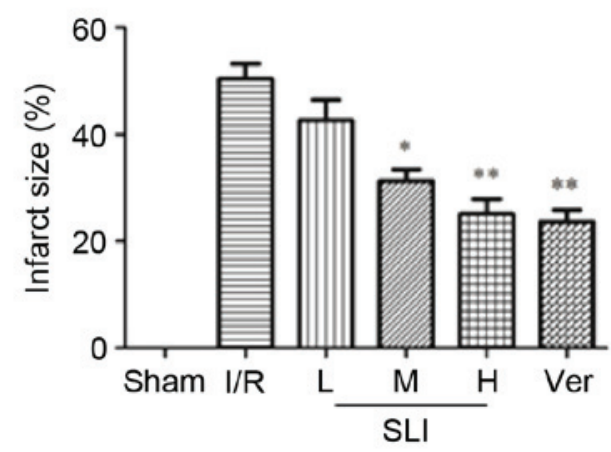

Figure 3. Effect of SLI on the size of myocardial infarction. (A) Images of triphenyl tetrazolium chloride dye staining of sections of the heart in various groups. (B) Quantification of infarct size was expressed as the percentage of the area at risk. Data are presented as the mean \pm standard deviation $(n=8$; ${ }^{*} \mathrm{P}<0.05,{ }^{* *} \mathrm{P}<0.01$ vs. I/R group). SLI, Salvia miltiorrhiza and ligustrazine injection; I/R, ischemia/reperfusion; L-, low-; M-, medium-; H-, high-; Ver, verapamil.

$(\mathrm{P}<0.05$ and $\mathrm{P}<0.01$; Fig. 2A, C and $\mathrm{D})$ and significantly increased the levels of SOD compared with rats in the I/R group $(\mathrm{P}<0.05 ; \mathrm{P}<0.01 ;$ Fig. $2 \mathrm{~B})$. These findings suggested that SLI may protect the heart against I/R-induced myocardial injury in vivo.

Effect of SLI on myocardial infarct size in $I / R$ rats. The ratios of infarct areas are shown in Fig. 3. MI was not observed in the hearts of the sham group, as expected. The ratio of infarct size was $50.24 \pm 9.51,31.87 \pm 3.75,42.63 \pm 8.56$ and $28.67 \pm 7.55 \%$ in the I/R, L-SLI, M-SLI and H-SLI groups, respectively. The M-SLI and H-SLI pretreatment groups exhibited significantly reduced infarct size compared with the $\mathrm{I} / \mathrm{R}$ group $(\mathrm{P}<0.05$ and $\mathrm{P}<0.01$, respectively; Fig. 3B). No significant difference was observed between the L-SLI and I/R groups (Fig. 3B).

Histological effect of SLI on the myocardium in I/R rats. The structure of the myocardium in the sham group was normal, with uniform cytoplasmic staining, consistent round or oval shape of cells and nuclear chromatin was uniformly distributed (Fig. 4A). The I/R group exhibited significant cardiomyocyte damage with extensive edema, myonecrosis and inflammatory cell infiltration. The nuclei were also chipped and dissolved (Fig. 4B). Pretreatment with H-SLI and Ver protected the cardiomyocytes from damage compared with the I/R group, as myofibril loss and inflammatory cell infiltration were decreased (Fig. 4E and F).

Effects of SLI on cell viability. Cell viability was assessed using a CCK-8 assay. The viability of $\mathrm{H} 9 \mathrm{C} 2$ cells incubated with SLI at various concentrations for $2 \mathrm{~h}$ was determined (Fig. 5). SLI concentrations between 0.01 and $1 \mu \mathrm{M}$ did not significantly affect cell viability (Fig. 5A). Conversely, 10 and $100 \mu \mathrm{M}$ SLI significantly reduced cell viability compared with the control group ( $\mathrm{P}<0.01$; Fig. 5A) Pretreatment with SLI reduced $\mathrm{H} / \mathrm{R}$-induced damage in $\mathrm{H} 9 \mathrm{C} 2$ cells, and significantly increased cell viability $(\mathrm{P}<0.05$ and $\mathrm{P}<0.01$; Fig. $5 \mathrm{~B})$. Therefore, $0.1 \mu \mathrm{M}$ SLI was used in subsequent in vitro experiments. 


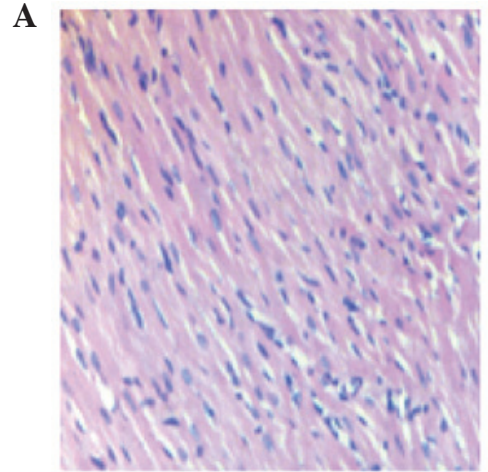

D

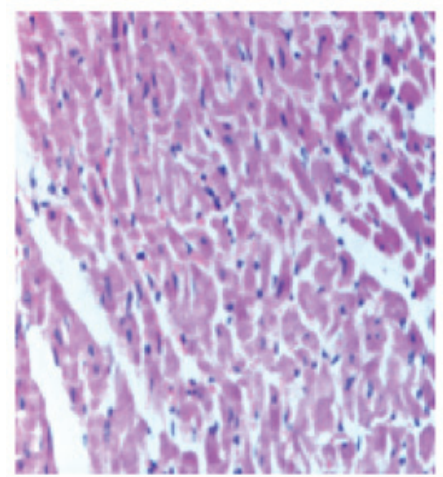

B

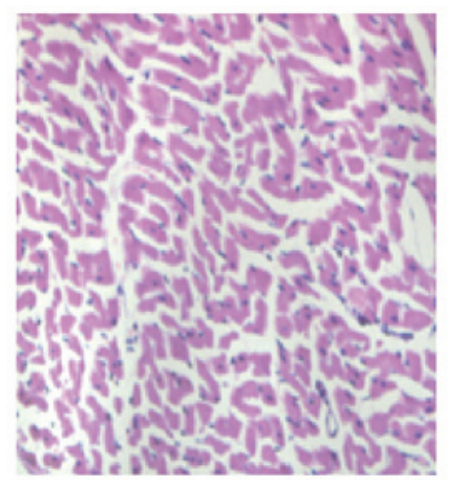

$\mathbf{E}$

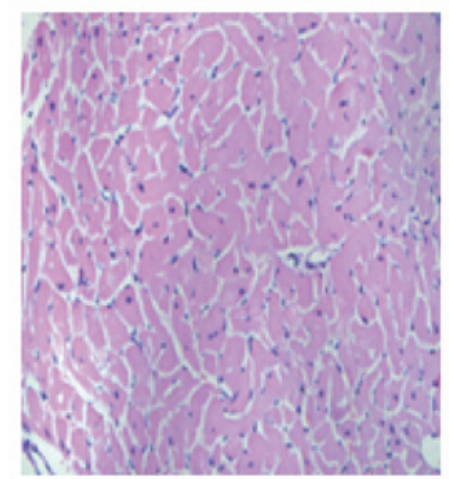

C

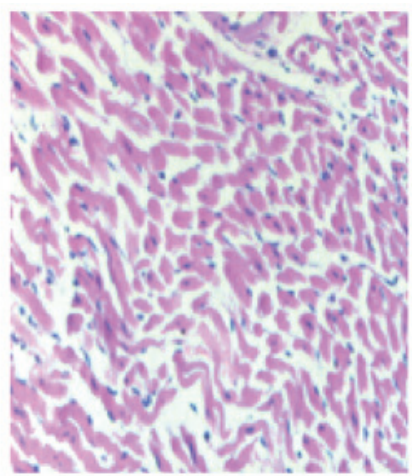

$\mathbf{F}$

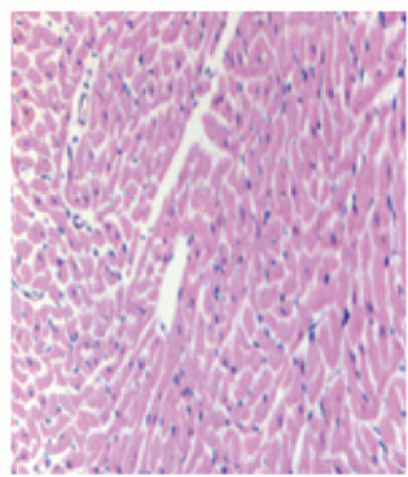

Figure 4. Microscopy images of rat myocardial tissue in response to various treatments. The myocardial tissue was stained with hematoxylin-eosin in the (A) sham group, where rats exhibited normal myocardial histology, clear transverse striations and no inflammatory cell infiltration, (B) the I/R group that exhibited evident swelling of myocardial cells, myonecrosis and inflammatory cell infiltration, (C) the L-SLI group that exhibited myocardial cell swelling degeneration, unclear arrangement, clear transverse striations and large numbers of inflammatory cells, (D) the M-SLI group that exhibited myocardial cell swelling, slight degeneration, unclear horizontal striations and reduced infiltration of inflammatory cells, (E) the H-SLI group had normal structure, clear transverse striations and few invasive inflammatory cells, and (F) the verapamil group with normal arrangement of cells, clear transverse striations and few invasive inflammatory cells. Magnification, x400. SLI, Salvia miltiorrhiza and ligustrazine injection; I/R, ischemia/reperfusion; L-, low-; M-, medium-; H-, high-.

A

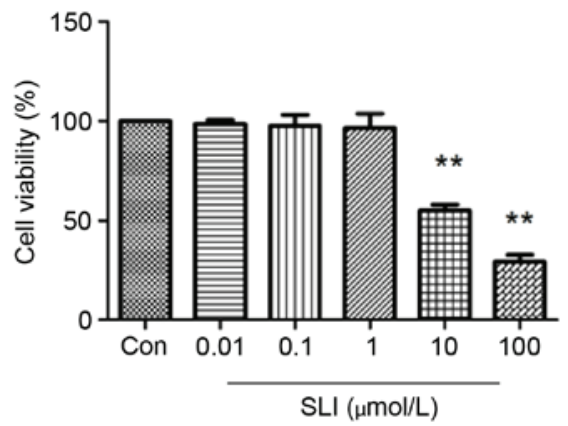

B

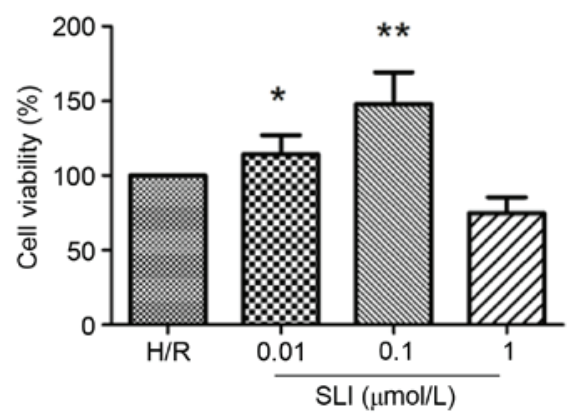

Figure 5. Effect of SLI on the cell viability of $\mathrm{H} 9 \mathrm{C} 2$ cells. The viability of cells with (A) SLI treatment alone (0.01-1 $\mu \mathrm{mol} / \mathrm{l})$ for $24 \mathrm{~h}$ was determined using an Cell Counting Kit-8 assay following treatment. No effect on $\mathrm{H} 9 \mathrm{C} 2$ viability was observed; however, when the concentration was increased between 10 and $100 \mu \mathrm{mol} / 1$ it became cytotoxic to H9C2 cells (B) Cell viability was determined using an Cell Counting Kit-8 assay following H/R. Data are expressed as the mean \pm standard deviation $\left({ }^{*} \mathrm{P}<0.05\right.$ and ${ }^{* *} \mathrm{P}<0.01$, vs. control or $\mathrm{H} / \mathrm{R}$ ). $\mathrm{H} / \mathrm{R}$, hypoxia/reoxygenation; SLI, Salvia miltiorrhiza and ligustrazine injection.
SLI increases Bcl-2 and decreases Bax and caspase-3 expression levels. Bcl-2 and Bax are important for mediating cell survival or death following apoptotic stimuli. As presented in Fig. 6A, pretreatment with $0.1 \mu \mathrm{M}$ SLI increased the expression of $\mathrm{Bcl}-2$ and inhibited the expression of Bax, resulting in a significantly increased Bcl-2/Bax ratio compared with the $\mathrm{H} / \mathrm{R}$ group $(\mathrm{P}<0.01)$. The caspase family of proteins are apoptosis regulators. Caspase- 3 is an important mediator for apoptosis and may be considered as a marker of H/R apoptosis. As presented in Fig. 6B, H/R significantly increased the activity of caspase- 3 compared with the control group $(\mathrm{P}<0.05)$, whereas $0.1 \mu \mathrm{M}$ SLI significantly reduced the levels of caspase- 3 compared with the $\mathrm{H} / \mathrm{R}$ group $(\mathrm{P}<0.01)$.

SLI increases the phosphorylation of Akt and eNOS. The molecular mechanism underlying SLI-mediated cardioprotection was investigated by western blot analysis. It has been previously reported that eNOS is a substrate for Akt and NO production following Akt-induced eNOS phosphorylation. The protein expression levels of p-Akt/Akt and p-eNOS in $\mathrm{H} 9 \mathrm{C} 2$ cells were determined following induction of H/R. SLI pretreatment significantly increased the expression levels of p-Akt and p-eNOS compared with the H/R group $(\mathrm{P}<0.01$; Fig. 7). Simultaneously, the Akt inhibitor, LY294002 significantly reduced the ratio of p-Akt/Akt compared with the SLI group $(\mathrm{P}<0.05 ;$ Fig. $7 \mathrm{~A})$. No significant difference was identified in the total protein expression levels of Akt and eNOS. These 
A
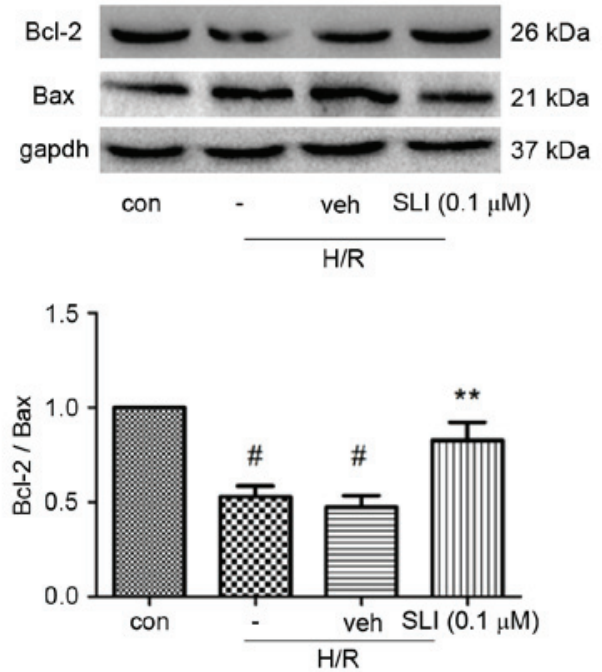

B
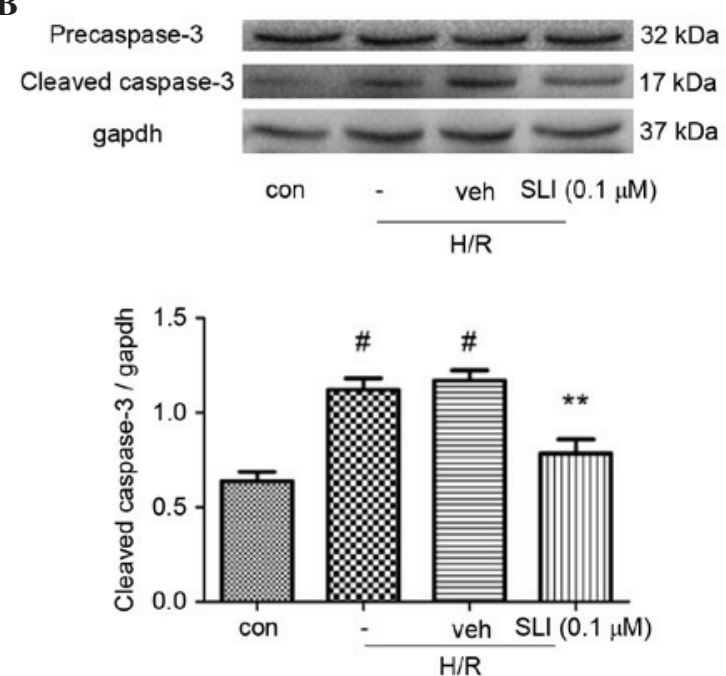

Figure 6. Effect of SLI on Bcl-2, Bax, caspase-3 levels following H/R injury. The protein expression levels of Bcl-2, Bax, caspase-3 subsequent to H/R injury were assessed by western blotting. (A) Pretreatment with $0.1 \mu \mathrm{M}$ SLI increased the expression of Bcl-2 and inhibited the expression of Bax; therefore, increased the Bcl-2/Bax ratio compared with the H/R group. (B) Treatment with $0.1 \mu \mathrm{M}$ SLI attenuated the levels of activated caspase-3 compared with the H/R group. Data are presented as the mean \pm standard deviation ( ${ }^{\#} \mathrm{P}<0.05$ vs. control group; ${ }^{* *} \mathrm{P}<0.01 \mathrm{vs}$. H/R group). H/R, hypoxia/reoxygenation; SLI, Salvia miltiorrhiza and ligustrazine injection; Bcl-2, B-cell lymphoma-2; Bax, Bcl-2-associated X protein; veh, vehicle; con, control.

A
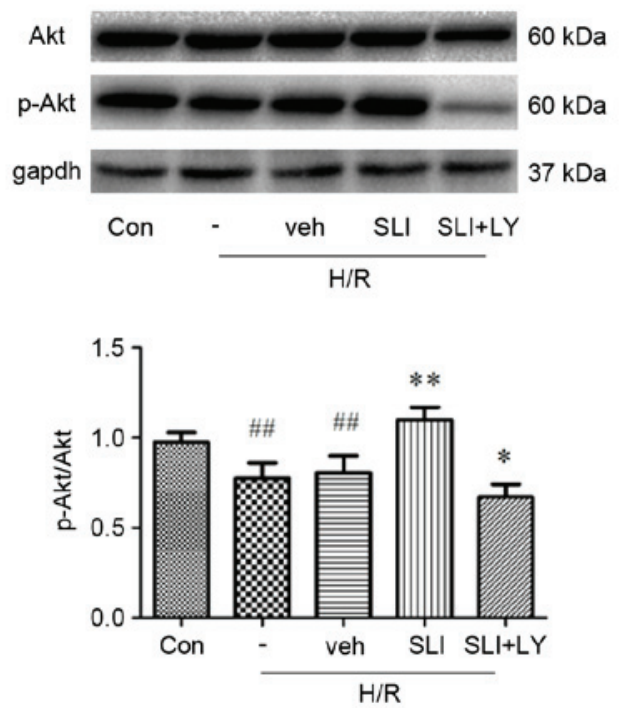

B
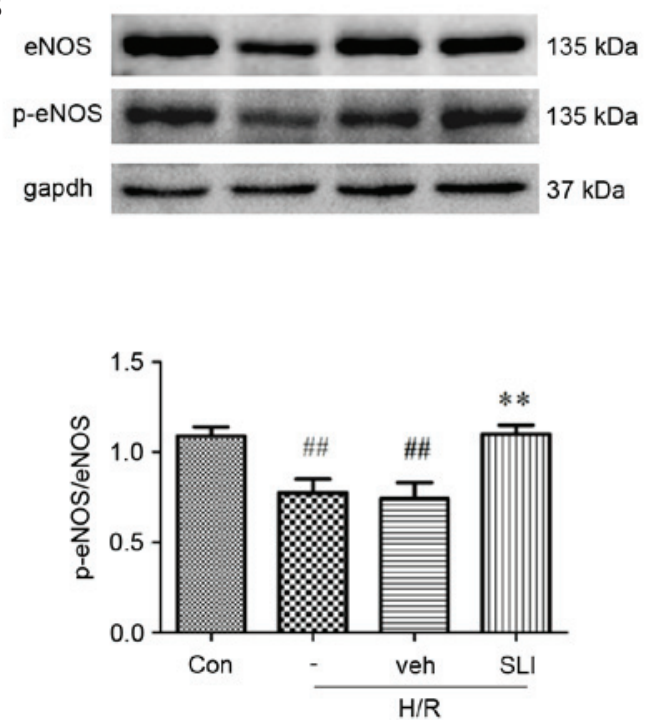

Figure 7. Effect of SLI on Akt and eNOS levels. The protein expression levels of Akt and eNOS was determined by western blot analysis following H/R injury. Pretreatment of 0.1 uM SLI improved the ratio of (A) p-Akt/Akt and (B) p-eNOS/eNOS. The Akt inhibitor, LY294002, significantly inhibited the ratio of p-Akt/Akt. Data are presented as the mean \pm standard deviation $\left({ }^{\# \#} \mathrm{P}<0.05\right.$ vs. control group; ${ }^{*} \mathrm{P}<0.01$ vs. SLI group; ${ }^{* *} \mathrm{P}<0.01$ vs. H/R group). H/R, hypoxia/reoxygenation; SLI, Salvia miltiorrhiza and ligustrazine injection; p-Akt, phosphorylated Akt serine/threonine kinase; p-eNOS, phosphorylated-endothelial nitric oxide synthase; veh, vehicle; con, control.

findings demonstrated that SLI may activate Akt and induce p-eNOS expression in cells where H/R is induced (Fig. 7).

\section{Discussion}

The present study evaluated the effects of SLI on myocardial $\mathrm{I} / \mathrm{R}$ injury in a rat model and $\mathrm{H} / \mathrm{R}$ injury in a rat myocardium cell line. The present study determined that SLI attenuated the effects of $\mathrm{I} / \mathrm{R}$ injury in vivo and $\mathrm{H} / \mathrm{R}$ injury in vitro.

To evaluate the effect of SLI on cardiac dysfunction following myocardial ischemia, hemodynamic parameters such as LVSP, LVDP and \pm dp/dt max were recorded. In the present study, the I/R injury group exhibited significant cardiac dysfunction, with reduced LVSP, LVDP and \pm dp/dt max. Pretreatment with SLI significantly reduced the changes in LVSP, LVDP and \pm dp/dt max following the induction of I/R. Therefore, SLI may ameliorate heart function impairment due to I/R injury. Cytosolic enzymes, including LDH and CK, may be used as diagnostic markers of myocardial ischemia injury, as they are released from the damaged myocardial tissues into the blood serum when cell membrane was induced to permeate or rupture. Therefore, $\mathrm{LDH}$ and $\mathrm{CK}$ levels in blood serum reflected changes in membrane integrity and the extent of myocardial injury. The present study determined that 
pretreatment with SLI reduced the elevated blood serum levels of LDH and CK induced by I/R injury, suggesting that SLI may reduce cell membrane damage in myocardial ischemic injury.

A previous study reported that oxidative stress is the primary contribution to myocardial I/R injury (19). Overproduction of reactive oxygen species may also result in lipid peroxidation process. SOD is often the primary mediator of oxygen free radicals due to its ability to reduce the production of free radicals and alleviate $H / R$ injury in myocardial cells. MDA is a terminal product of lipid peroxidation and its concentration in blood serum may reflect the extent of myocardial injury (1). In the present study, pretreatment with SLI significantly reduced MDA levels and increased SOD activity. The current findings indicated that SLI may enhance the elimination of oxygen free radicals and reduce myocardial injury.

Calculation of the MI area and the myocardial pathomorphism are the most intuitive methods of assessing myocardial damage. Myocardial injury may lead to membrane damage of cardiomyocytes with extensive edema, myonecrosis, infiltration of inflammatory cells, disrupted cristae, chromatin condensation, cytoplasmic vacuoles and loss of myofibrils. SLI pretreatment may reduce cardiomyocyte membrane damage induced during MI with smaller areas of myofibril loss and reduced inflammatory cell infiltration, swelling and vacuolation of mitochondrial cristae and decreased the infarct size. In order to determine the mechanism behind the positive SLI effects in H9C2 cells. Akt was identified as the primary target enzyme; therefore, phosphorylation and expression of Akt may influence protein synthesis of apoptotic factors, including inhibiting the activation of caspase- 3 and increasing the Bcl-2/Bax ratio. Conversely, previous studies have determined that phosphorylation of Akt may activate eNOS, which is the downstream target of RISK pathways, and may induce p-eNOS function as a cardiovascular protective molecule (20-22). Further investigation is required in order to fully elucidate the molecular mechanisms behind the protective effect of SLI on I/R and $\mathrm{H} / \mathrm{R}$-damaged myocardial tissues.

To the best of our knowledge, the present study is the first to demonstrate that SLI exerted cardioprotective effects in rats with myocardial I/R injury via the regulation of myocardial enzymes and increasing the levels of enzymes responsible for eliminating oxygen free radicals. Additionally, SLI pretreatment may inhibit cell apoptosis via activation of the Akt-eNOS signaling pathway.

\section{References}

1. Ren-an Q, Juan L, Chuyuan L, Wenjuan F, Chunyan H, Xuemei Y, Lin $\mathrm{H}$ and Hong N: Study of the protective mechanisms of Compound Danshen Tablet (Fufang Danshen Pian) against myocardial ischemia/reperfusion injury via the Akt-eNOS signaling pathway in rats. J Ethnopharmacol 156: 190-198, 2014

2. Lopez AD and Murray CC: The global burden of disease, 1990-2020. Nat Med 4: 1241-1243, 1998.
3. Shimokawa $\mathrm{H}$ and Yasuda S: Myocardial ischemia: Current concepts and future perspectives. J Cardiol 52: 67-78, 2008.

4. Morgan EN, Boyle EM Jr, Yun W, Kovacich JC, Canty TG Jr, Chi E, Pohlman TH and Verrier ED: Platelet-activating factor acetylhydrolase prevents myocardial ischemia-reperfusion injury. Circulation 100 (19 Suppl): II365-II368, 1999.

5. Li SY, Wang XG, Ma MM, Liu Y, Du YH, Lv XF, Zhou JG, Tang YB and Guan YY: Ginsenoside-Rd potentiates apoptosis induced by hydrogen peroxide in basilar artery smooth muscle cells through the mitochondrial pathway. Apoptosis 17: 113-120, 2012.

6. Wang Y, Li X, Wang X, Lau W, Wang Y, Xing Y, Zhang X, Ma X and Gao F: Ginsenoside Rd attenuates myocardial ischemia/reperfusion injury via Akt/GSK-3 $\beta$ signaling and inhibition of the mitochondria-dependent apoptotic pathway. PLoS One 8: e70956, 2013.

7. Zhu J, Qiu Y, Wang Q, Zhu Y, Hu S, Zheng L, Wang L and Zhang Y: Low dose cyclophosphamide rescues myocardial function from ischemia-reperfusion in rats. Eur J Cardiothorac Surg 34: 661-666, 2008.

8. Ji XY, Tan BK and Zhu YZ: Salvia miltiorrhiza and ischemic diseases. Acta Pharmacol Sin 21: 1089-1094, 2000.

9. Dong ZT and Jiang WD: Effect of danshensu on isolated swine coronary artery perfusion preparation (author's transl). Yao Xue Xue Bao 17: 226-228, 1982 (In Chinese).

10. Lam FF, Yeung JH, Chan KM and Or PM: Relaxant effects of danshen aqueous extract and its constituent danshensu on rat coronary artery are mediated by inhibition of calcium channels. Vascul Pharmacol 46: 271-277, 2007.

11. Wu L, Qiao H, Li Y and Li L: Protective roles of puerarin and Danshensu on acute ischemic myocardial injury in rats. Phytomedicine 14: 652-658, 2007.

12. Wong KL, Wu KC, Wu RS, Chou YH, Cheng TH and Hong HJ: Tetramethylpyrazine inhibits angiotensin II-increased NAD(P)H oxidase activity and subsequent proliferation in rat aortic smooth muscle cells. Am J Chin Med 35: 1021-1035, 2007.

13. Feng L, Ke N, Cheng F, Guo Y, Li S, Li Q and Li Y: The protective mechanism of ligustrazine against renal ischemia/reperfusion injury. J Surg Res 166: 298-305, 2011.

14. Zhang XP, Wang C, Wu DJ, Ma ML and Ou JM: Protective effects of ligustrazine, kakonein and Panax notoginsenosides on multiple organs in rats with severe acute pancreatitis. Methods Find Exp Clin Pharmacol 32: 631-644, 2010.

15. Huang WD, Yang YF, Chen JW and Zhu BH: Synergism of salviamiltiorriza bunge and tetramethylpyrazine on cardiovascular system in rat. Chinese Pharmacological Bulletin: 432-436, 2013. (In Chinese)

16. Buerke M, Murohara T, Skurk C, Nuss C, Tomaselli K and Lefer AM: Cardioprotective effect of insulin-like growth factor I in myocardial ischemia followed by reperfusion. Proc Natl Acad Sci USA 92: 8031-8035, 1995.

17. Miki T, Miura T, Tsuchida A, Nakano A, Hasegawa T, Fukuma T and Shimamoto K: Cardioprotective mechanism of ischemic preconditioning is impaire by postinfarct ventricular remodeling through angiotensin II type 1 receptor activation. J Cardiol 37: 112-113, 2001 (In Japanese).

18. Oron U, Yaakobi T, Oron A, Mordechovitz D, Shofti R, Hayam G, Dror U, Gepstein L, Wolf T, Haudenschild C and Haim SB: Low-energy laser irradiation reduces formation of scar tissue after myocardial infarction in rats and dogs. Circulation 103: 296-301, 2001.

19. Kaminski KA, Bonda TA, Korecki J and Musial WJ: Oxidative stress and neutrophil activation-the two keystones of ischemia/reperfusion injury. Int J Cardiol 86: 41-59, 2002.

20. Schulz R, Kelm M and Heusch G: Nitric oxide in myocardial ischemia/reperfusion injury. Cardiovasc Res 61: 402-413, 2004.

21. Thirunavukkarasu M, Penumathsa SV, Koneru S, Juhasz B, Zhan L, Otani H, Bagchi D, Das DK and Maulik N: Resveratrol alleviates cardiac dysfunction in streptozotocin-induced diabetes: Role of nitric oxide, thioredoxin, and heme oxygenase. Free Radic Biol Med 43: 720-729, 2007.

22. Vinten-Johansen J, Zhao ZQ, Jiang R, Zatta AJ and Dobson GP: Preconditioning and postconditioning: Innate cardioprotection from ischemia-reperfusion injury. J Appl Physiol (1985) 103: $1441-1448,2007$. 